\title{
ALGEBRAIC SURFACES WITH REDUCIBLE BITANGENT AND OSCULATING HYPERPLANAR SECTIONS*
}

\author{
BY \\ MARIA CASTELLANI
}

The purpose of this investigation is to follow along certain lines the researches of the Italian geometric school on surfaces in hyperspace which have particular systems of reducible hyperplanar sections. In this paper two cases are discussed, first, surfaces which are cut by any bitangent hyperplane along reducible curves, second, surfaces which are cut by any osculating hyperplane along reducible curves. The present investigation may be considered as an extension of work done by Castelnuovo and Enriques. The results are obtained with the aid of algebraic geometry and projective differential geometry.

\section{PART I}

1. Reducible bitangent sections. In order to study what kind of hyperspatial, algebraic irreducible surfaces $F$, which are not cones, are cut by a bitangent hyperplane in reducible curves we choose the inductive method. We project such a surface from an arbitrary tangent plane and we obtain a surface cut by any tangent hyperplane in a reducible curve. This surface will be either a surface of Veronese of $S_{5}$ or a ruled surface.t

Let us suppose that $F$ is not cut by a tangent plane in a reducible curve, consequently $F$ is not a ruled surface or a surface of Veronese: the space $S_{r}$. to which $F$ belongs is of dimensions $r>5 t$ and the space of two arbitrary tangent planes should be an $S_{5}$. We introduce also the hypothesis that such an $S_{5}$ has no points of $F$ outside of the two established points.

Let us take an arbitrary tangent plane $\pi$. From $\pi$ we project $F$ upon an $S_{r-8}$, obtaining a surface $F^{\prime}$ which may be a simple or a multiple projection of $F$. We can show at once that the second case has to be excluded. In fact if the projection upon $F$ is doubled (the case may be easily

\footnotetext{
* Presented to the Society, May 2, 1925.

†F. Enriques, Sulla massima dimensione dei sistemi lineari di curve di dato genere appartenenti ad una superficie algebrica, Atti della Reale Accademia delle Scienze di Torino, vol. 29 (1893-94), pp. 279-280.

$\ddagger$ A. Terracini, Su due problemi concernenti la determinazione di alcune classi di superficie considerate da G. Scorza e da F. Palatini, Atti della Società dei Naturalisti e Matematici di Modena, ser. 5, vol. 6 (1921-1922).
} 
generalised) the space $S_{3}$, which projects $F$ from $\pi$, has to meet $F$ in two distinct points $Q_{1}$ and $Q_{2}$ whose projections upon $F^{\prime}$ have to be the same. Consequently the straight line through $Q_{1}, Q_{2}$ belongs to the $S_{3}$ determined by $\pi$ and one of the two points. We have $\infty^{2}$ pairs of points associated with the $\infty^{2}$ points of $F$. It follows that at least $\infty^{2}$ chords of $F$ meet $\pi$. But as the projections of $Q_{1}$ and $Q_{2}$ are the same point $Q^{\prime}$ of $F^{\prime}$, the tangent planes $\pi_{1}$ and $\pi_{2}$ respectively in $Q_{1}$ and $Q_{2}$ at $F$ have to project into the same tangent plane $\pi^{\prime}$ at $Q^{\prime}$ to $F^{\prime}$, therefore the two $S_{3}$ projecting the two points $Q_{1}$ and $Q_{2}$ coincide. The three tangent planes $\pi, \pi_{1}, \pi_{2}$ belong to the same $S_{3}$. When we take as the center of projection $\pi_{1}$ instead of $\pi$, the two tangent planes $\pi$ and $\pi_{2}$ are projected into the same plane $\pi^{\prime}$, but a tangent plane cannot have two distinct points of contact, so for the coincidence of the projections of the two planes $\pi$ and $\pi_{2}$, we have the coincidence of the projections of the two points $P$ and $Q_{2}$. Accordingly $P$ and $Q_{2}$ give one of the above considered $\infty^{2}$ chords of $F$, but the three points $P, Q_{1}, Q_{2}$ cannot be on the same straight line because each chord cannot cut the surface in three points. ${ }^{*}$ Therefore they give a plane $\sigma$. The plane $\sigma$ belongs to the $S_{3}$ which projects $Q_{1}$ from $\pi$, and to the $S_{3}$ which projects $P$ from $\pi_{1}$. Evidently the planes $\pi, \pi_{1}, \pi_{2}$ cut $\sigma$ in straight lines. Accordingly two tangent planes have a common point.

We arrive at the same conclusions if we take another pair of points, that are projected into the same point $\pi_{1}$, consequently two arbitrary tangent planes have a common point, and $F$ is either a surface of Veronese of $S_{3}$, or a ruled surface contrary to our hypothesis.t Therefore we can conclude that $F$ is projected from any tangent plane in a one-to-one way upon a surface $F^{\prime}$ of $S_{r--;}$, and accordingly the hyperplanar sections of $F^{\prime}$ are in one-to- one correspondence with the hyperplanar tangent sections of $F$.

So when the hyperplanar sections of $F$ have genus $p$, the hyperplanar sections of $F^{\prime}$ have genus $p-1$ because they correspond to the hyperplanar tangent sections of $F$. Noreover the hyperplanar tangent sections of $F^{\prime}$ correspond to the hyperplanar bitangent sections of $F$, and the former have to be reducible, because the latter are reducible.

Accordingly $F^{\prime}$ is either a ruled surface or a surface of Veronese of $S_{5}$. We shall study the two cases separately.

2. The surface $F^{\prime}$ is a ruled surface. As the surface $F^{\prime}$ has $\infty^{1}$ straight lines the surface $F$ has a pencil $(C)$ of rational curves $C$ cut in one variable

* E. Bertini, Introduzione alla Geometria Proiettiva degli Iperspazi, Pisa, Spoerri, 1907, pp. 194-195.

†P. Del Pezzo, Sulle superficie dell' $n^{m o}$ ordine immerse nello spazio di $n$ dimensioni, Rendiconti del Circolo Matematicu di Palermo, vol: 1 (1887), pp. 241-271. 
point by the $S_{r-1}$ of $S_{r}$ because the pencil $(C)$ is projected on $\infty^{1}$ straight lines of $F^{\prime}$.

The pencil $(C)$ may be irrational or rational.

$(a)$ Let us suppose $(C)$ irrational.

The pencil $(C)$ cannot change with the center $P$ of projection, because if $(C)$ changes there will be a continuum of irrational pencils and consequently upon each section of $F$ a continuum of irrational involutions. This is impossible because of a theorem of Castelnuovo.*

If the pencil $(C)$ is not variable any hyperplane through $\pi$ cuts any $C$ in several points, but under our assumption only one is variable, so apart from this one variable point, there are some fixed points on the plane $\pi$. The tangent plane $\pi$ has to meet all the curves $C$, and similarly for all the tangents $S_{2}$. But as the tangent planes do not cut $F$ in curves it follows that the tangent planes have to pass through at least one basis point of the infinite pencil $(C) .+$ Accordingly the projection of $F$ from an $S_{r-4}$ upon an $S_{3}$ should be a surface with all tangent planes having a point in common, therefore this surface is a cone and also $F$ is a cone contrary to our hypothesis.

Then $F$ is a surface with an $\infty^{1}$ of curves $C$ and the hyperplane through any tangent plane which does not meet them cuts them in only one point. Hence $F$ is a ruled surface.

(b) The pencil is rational.

The surface $F$ to which a rational pencil of rational curves belongs is rational according to a well known theorem of Noether $f$ Such a surface can be represented upon a plane with a system of curves in one-to-one correspondence with hyperplanar sections.

So, as we can show from the plane representation, any $S_{r-1}$ through $\pi$ (an arbitrary tangent plane) cutting $C$ in only one variable point, cuts $F$ in rational irreducible curves. Therefore $p-1=0$ and $p=1$.

If the surface $F$ is a surface with the hyperplanar sections of genus $p$ then, from a theorem of Castelnuovo $F$ has order $r \leqq 9$ and can be represented upon a plane by the system of cubics with $9-r$ simple points in common, or with the system of quartics with 2 double common points.

* G. Castelnuovo, Sulla linearità delle involuzioni piu volte infinite appartenenti ad una curva algebraica, Atti della Reale Accademia delle Scienze di Torino, vol. 28 (1892-93), pp. 727-738.

$\dagger$ G. Castelnuovo, Massima dimensione dei sistemi lineari di curve piane di dato genere, Annali di Matematica, ser. 2, vol. 18 (1890), pp. 119-128.

$\ddagger$ M. Noether, Ueber Flächen, welche Schaaren rationaler Curven besitzen, Math e m a tis ch $\mathrm{e}$ Annalen, vol. 3 (1871), pp.161-270.

$\S$ G. Castelnuovo, Sulle superficie algebriche le cui sezioni piane sone curve ellittiche, Rendiconti della Reale Accademia dei Lincei, ser. 4, vol. 3 (1894), pp. 59-61. 
But here we take only the first case into consideration, because the second gives a surface of the eighth order and second kind of $S_{8}$, which is projected by a tangent plane not into a ruled surface but into a surface of Veronese.

The cubics corresponding to the hyperplanar bitangent sections are the cubics with two common double points outside the common points of the system. Therefore the cubics are composed of conics of a system $\infty^{r-6}$ and with one common straight line joining the two points corresponding to the two points of contact of the bitangent hyperplanes.

When $r=9$ the hyperplanar bitangent sections of a surface $F$ are composed of curves of the third order and the sixth order. The first are common to all $\infty^{3}$ bitangent $S_{8}$ at a fixed point of $F$, the second are variable in an $\infty^{3}$ system with two base points.

3. Surface $F^{\prime}$ a surface of Veronese of $S_{5}$. Evidently as $r-3=5$, the surface $F$ belongs to an $S_{8}$ and it is a rational surface because it is in one-to-one correspondence with the surface of Veronese, and therefore $F$ can be represented upon a plane.

As in the above mentioned case, we arrive at the conclusion that the tangent sections of $F$ have genus $p-1=0$, because they are in correspondence with the hyperplanar rational sections of the surface of Veronese.

So also in this case the hyperplanar sections of $F$ have genus $p=1$.

Evidently $F$ is a surface of the eighth order and second kind in correspondence with the system of plane quartics with two common double points.*

The plane curves corresponding to the hyperplanar tangent sections of $F$ are quartics with two other double points. These quartics are composed of two conics of a pencil. Accordingly the bitangent sections of the $S_{r-1}$ cut $F$ in a pencil of curves of the fourth order of which the two points of contact are basis points.

Theorem I. The algebraic irreducible surfaces of $S_{r}(r>5)$, not cones, which any bitangent hyperplane cuts in reducible curves are the ruled surfaces and the surfaces with hyperplanar sections of genus $p=1$.

\section{PART II}

1. Reducible osculating sections.t We shall consider only the fundamental types of surfaces $F$, because many difficulties arise when we leave the neighborhood of the first order of a point and enter into the neighborhoods of a higher order. So we make the following assumptions:

* Del Pezzo, Sulle superficie dell' $n^{m o}$ ordine immerse nello spazio di $n$ dimensioni, Rendiconti del Circolo Matematico di Palermo, vol. 1 (1887), pp. 241-271.

$\uparrow$ The osculating sections are given by the hyperplanes through the minimum osculating space. If we take the notation of Bompiani we represent this as $S(2)$. This means that we have to take the derived points of the first and second order of a fixed point. All the planes osculating the curves through a fixed point lie in an $S(2)$. The $S(2)$ is in general 
(a) $F$ is an algebraic irreducible surface, not a cone, of a convenient $S_{r}$; (b) the osculating $S_{r-1}$ have to cut $F$ in curves with an ordinary triple point at the point of osculation;* apart from such a point they may not necessarily contain another point of osculation or any point of contact and may not have a common space; (c) there are $\infty^{2} S(2)$-one for each point of the surface-the $S(2)$ are $S_{5}$ and they do not cut the surface in reducible curves when they cut $F ;(d)$ two tangent planes at two consecutive points give an $S_{4}$; these $S_{5}$ do not cut the surface in curves and do not have a common space.

The first type of surface which satisfies these conditions is the surface with reducible tangent sections, hence for $r>5$ the ruled surfaces. $t$

If we consider the osculating $S_{r-1}$ at any non-singular point $P$ of $F$ these osculating $S_{r-1}$ have for definition a common $S(2)$. With our hypothesis this common space is $S_{5}$, accordingly such $S_{r}$ form a linear system $\infty^{r-6}$ and in such a system the curves which they cut on $F$ are variable. $\S$

But for our purpose such curves have to be reducible. So, by a theorem of Enriques, either they are composed of several curves of a rational or irrational pencil of which only one goes through an arbitrary point of $F$, or they are composed of common curves and others, reducible or not, of a system $\infty^{r-6}$.

Let us study the two cases separately.

2. Osculating sections at one point are composed of curves of a pencil. The osculating hyperplanes at a point $P$ of $F$ cut the surface in curves composed of some curves of a pencil $(C)$. We shall show that the pencil $(C)$ varies with $P$, that all the pencils thus obtained lie in a system $\infty^{2}$ and that the curves $C$ are of the third order and therefore the dimension $r$ of the space is $\leqq 9$.

In fact if $(C)$ does not vary with $\bar{P}$, either all the osculating sections have common fixed curves, which is contrary to our hypothesis, or two an $S_{5}$ (Bompiani, Sopra alcune estensioni dei teoremi die Meusnier e di Eulero, Atti della Reale Accademia delle Scienze di Torin o, vol. 48 (1912-1913), p. 393). The osculating hyperplane cuts a surface in a curve with at least a triple point at the point of osculation.

* Del Pezzo, Sulle superficie dell' $n^{m o}$ ordine immerse nello spazio di $n$ dimensioni, Rendiconti del Circolo Matematico di Palermo, vol. 1 (1887), pp. 241-271.

$\dagger$ E. Bompiani, Determinazione delle superficie integrali di un sistema di equazioni a derivate parziali lineari ed omogenee, Atti della Reale Accademia delle Scienze di Torino, vol. 52 (1919-1920), pp. 614-615.

M. Castellani, Sulle superficie $i$ cui spazi osculatori sono biosculatori, Rendiconti della Reale Accademia dei Lincei, ser. 4, vol. 29 (1922), pp. 347-350.

$\ddagger$ F. Enriques, Sulla massima dimensione dei sistemi lineari di curve di dato genere appartenenti ad una superficie algebrica, Atti della Reale Accademia delle Scienze di Torino, vol. 29 (1893-1894), pp. 279-280.

$\S$ E. Bertini, Introduzione alla Geometria Proiettiva degli Iperspazi, p. 26. 
curves $C^{\prime}$ go through each point of the surface, which is contrary to the theorem of Enriques, and therefore the pencil $P$ varies with the point $P$. When the point $P$ varies the pencil $(C)$ gives a continuous system at least $\infty{ }^{2}$.

Such a system cannot have basis points, because by our hypothesis the osculating hyperplanes cannot have a common space, therefore the curves $C$ cut each other in $\nu$ variable points.

If $\nu \geqq 2$ every pencil $(C)$ would have $\nu \geqq 2$ basis points, coincident or not, and the osculating $S_{r-1}$ at $P$ of $F$ would have points of contact or osculation not at $P$, or a point of osculation of higher order in $P$. But such a case is contrary to our hypothesis, so we may conclude that the curves $C$ of $(C)$ cut each other only in $P$ where they have a simple intersection.

The curves $C$ of the system described by the pencil $(C)$ cut each other only in one variable point, accordingly through any two points of $F$ only one curve of the system passes. By a theorem of Enriques* it is a linear system $\infty^{2}$. The curves $C$ are rational because the characteristic series on any of them is a $g_{1}^{1}$; therefore it is easy to show that the curves $C$ are rational curves of the third order of $S_{3}$.

In fact let us take an arbitrary curve $C$ and an $S_{r-1}$ which osculates the surface in a point not belonging to $C$. Such an $S_{r-1}$ cuts the surface in three curves and as each of these meets $C$ in one point only, the $S_{r-1}$ intersects $C$ in three points and accordingly the curves $C$ are curves of the third order. Since they are rational and not plane, they are cubics of $S_{3} . t$

The surface $F$ to which an $\infty^{2}$ of curves of the third order belong is the rational, normal surface of $S_{9}$ which can be represented on a plane by the system of plane cubics-or a projection.

3. Osculating sections at one point have a common curve. Let us now study the case in which the osculating hyperplanes at a non-singular point $P$ of a surface $F$ cut the surface in a fixed irreducible curve $T$ and in curves $C$ of a system.

The fixed curve $T$ evidently belongs to the $S(2)$ osculating the surface $F$ at the point $P$. Therefore our problem is now to study the maximum space of curves that the $\infty^{2}$ osculating $S(2)$ cut upon a surface.

We have to take into consideration two cases: $(a)$ there are $\infty^{1}$ curves on $F$; (b) there are $\infty^{2}$ curves on $F$.

* F. Enriques, Ricerche di geometria sopra una superficie algebrica, Memorie della Reale Accademia delle Scienze di Torino, ser. 2, vol. 44 (1894), pp. 179-232.

$\dagger$ A surface with $\infty^{n}$ curves of $S_{n}$ belongs to an $S_{r}$ where $r \leqq n(n+3) / 2$; G. Castelnuovo, Sulle superficie algebriche le cui sezioni piane sono curve ellittiche, Rendiconti della Reale Accademia dei Lincei, ser. 4, vol. 3 (1894), pp. 59-61; E. Bompiani, Proprietà differenziali caratteristiche di enti algebrici, Memorie della Reale Accademia dei Lincei, ser. 4, vol. 29 (1922). 
(a) Let us begin by supposing that they are curves of $S_{5}$. In such a case the osculating $S(2)$ at any point of $T$ are the $S_{5}$ of $T$, so such an $S_{5}$ osculates $T$ of the surface $F$ at all its points. Accordingly in this space there will be $T$ and two other curves $T^{\prime}$ and $T^{\prime \prime}$ infinitely near to $T^{\prime}$. If we repeat the same process to $T^{\prime}$ we find that in the $S_{5}$ of $T$ there will be $T^{\prime}, T^{\prime \prime}, T^{\prime \prime \prime}$ and in the same way we can conclude that the $T^{\prime}$ belong to such an $S_{5}$ and therefore the entire surface $F$.

When the curves $T^{\prime}$ belong to an $S_{4}$ it is easy to find a similar result. In fact the $S$ of $T^{\prime}$ and the tangent at the point $P^{\prime}$-infinitely near $P$ to its curve $T^{\prime}$ are by definition in the same $S_{5}(2)$-osculating $F$ in $P$. Then when $P$ describes $T^{\prime}$ we find that the $S_{4}$ of $T^{\prime}$ is met by all tangents at any point of $T^{\prime}$ because $P^{\prime}$ describes $T^{\prime}$. Consequently $T$ and $T^{\prime}$ are in the same $S_{5}$, but such an $S_{5}$ may be fixed with the $S_{4}$ of $T$ and any point of $T^{\prime}$. The same property holds for $T^{\prime}$ and $T^{\prime \prime}$; as a point of $T^{\prime \prime}$ is in the osculating $S(2)$ at $P$ we find that this $S(2)$ osculates $T^{\prime}$ in all its points. As in the case just considered we find that $F$ lies in such an $S_{5}$. When the $F$ lies in $S_{3}$ we have several cases.

When two $S_{3}$ infinitely near together belong to an $S_{7}$, as any $S(2)$ osculating $F$ at any point of $T$ may be given by the $S_{3}$ of $T$ and a tangent at $T^{\prime}$ in the point $P^{\prime}$ infinitely near to $P$ not on $T$ we have the $S_{7}$ of two infinitely near curves which osculate $F$ at any point of $T$, and consequently $F$ is a surface of $S_{7}$. We can arrive at the same results if we suppose two curves $T$ and $T^{\prime}$ in $S_{6}$ or $S_{5}$.

If instead two curves are in an $S_{4}$, by the theorem of Bompiani* it follows that $F$ is a surface with $\infty^{1}$ curves $T$ in $S_{3}$ of a developable surface. Along such curves the $S_{5}^{r}$ osculate $T$ in all points. The $T$ in $S_{3}$ gives results contrary to our fundamental hypothesis.

The case of plane curves is always possible.

(b) When the $T$ are $\infty^{2}$ the $S_{r}$ have as dimensions $r<8$. In order to show this property we take into consideration some results of algebraic geometry.

Let us take an arbitrary point $P$ of $T$. The $S(2)$ osculating the surface at $P$ cuts $F$ in a curve $T$. Any $S_{r-1}$ through such an $S(2)$ cuts the surface in $T$ and in another curve $C$ of a system $\infty^{r-6}$. By our previous hypothesis this section does not have a point of contact or osculation except at the fixed point $P$ and the curve $C$ will not meet $T$ in a variable point. Accordingly when we take the curves $C$ for any point of $T$, any one of $C$ is reduced to $T$ and in general to another curve of a system $\infty^{r-7}$. The existence of such a residual curve is not needed, only we have to observe

* E. Bompiani, Determinazione delle superficie integrali di un sistema di equazioni a derivate parziali lineari el omogonee, Atti della Reale Accademia delle Scienze di Torino, vol. 52 (1919-1920), pp. 614-615. 
that from the system of the $\infty^{r-6} S_{r-1}$ osculating at $P$ we have taken a system $\infty^{r-7}$ of hyperplanes tangential to $F$ at every point of $T$. But by a well known theorem* a linear system $\infty^{r-7}$ of hyperplanes has an $S_{6}^{\prime}$ in common, so the $S_{6}^{\prime}$ common to all the above mentioned hyperplanes is tangent to $F$ at every point of $T$. If the curve $C$ by the condition imposed is reduced to two curves like $T$ we have the $S_{6}$ osculating $F$ in all points $T$ so in such an $S_{6}$ it would be the $\infty^{1} T$ and the entire surface would belong to an $S_{6}$.

Now let us consider the first case and take such an $S_{6}$ and an $S(2)$ osculating $F$ at any point of $T$, for instance at $Q$. In such an $S(2)$ there are two tangent planes at two points of the curve $T$ infinitely near together and by our hypothesis two infinitely near tangent planes give an $S_{4}$. The $S_{6}$ above considered osculates $F$ at every point of $T$. We observe that the curves $T$ are $\infty^{2}$ and we have $\infty^{1}$ curves $T$ for any point of $F$, so the curves $T$ meet each other in at least one variable point.

Any $S(2)$ osculating $F$ in a point other than the points $P$ and $Q$ has in common with this $S_{7}$ the $S_{4}$ of two tangent planes at two consecutive points of $T$ and the point of intersection $R$ of curves cut by the $S(2)$ osculating $Q$. Also the $S(2)$ that we have now taken in consideration belongs to such an $S_{7} . \quad R$ does not belong to the $S_{4}$ of two consecutive tangent planes above considered because if this were so it would also be true for any $S_{4}$ tangent to two consecutive points of $T$. This means that either all the $S_{4}$ have a common point or as $P$ describes $T$, there are $\infty^{1} R$. Both cases are contrary to our hypothesis. So the point $R$ and such an $S_{4}$ give an $S_{5}(2)$ osculating $F$ in a point of $T$. Accordingly such an $S(2)$ is in the $S_{7}$ above considered. Consequently the $S_{7}$ contains all the $S(2)$ osculating $T$ at every point $Q$, so that it contains $\infty^{1}$ curves and obviously the entire surface.

THeOREM II. Two classes of algebraic surfaces (not cones) are cut in reducible curves by the osculating $S_{r-1}$ when the $S_{r-1}$ have neither a common space nor points of osculation outside the fixed point; when the $S(2)$ are $\infty^{2} S_{5}$ which do not cut the surface in reducible curves and when the $S_{4}$ of two consecutive tangent planes do not cut the surface:

(1) the surfaces of $S_{r}(r>7)$ cut in curves by the $S(2)$ are ruled surfaces and surfaces with $\infty^{1}$ plane curves;

(2) the surfaces of $S_{r}(r>6)$ which the $S(2)$ do not cut are the rational normal surfaces of $S_{9}$ with $\infty^{2}$ curves of the third order or a projection.

* E. Bertini, Introduzione alla Geometria Proiettiva degli Iperspazi, p. 26.

Bryn Mawr College,

Bryn Mawr, Pa. 